\title{
Indirect revascularization with the dural inversion technique for pediatric moyamoya disease: 20-year experience
}

\author{
*Nisha Gadgil, MD, ${ }^{1}$ Sandi Lam, MD, MBA, ${ }^{1}$ Monika Pyarali, BS, ${ }^{1}$ Michael Paldino, MD, ${ }^{2}$ \\ I-Wen Pan, PhD, ${ }^{1}$ and Robert C. Dauser, MD'1 \\ 1Department of Neurosurgery, Division of Pediatric Neurosurgery, Baylor College of Medicine/Texas Children's Hospital; and \\ 2Department of Radiology, Texas Children's Hospital, Houston, Texas
}

\begin{abstract}
OBJECTIVE Numerous surgical procedures facilitate revascularization of the ischemic brain in patients with moyamoya disease. Dural inversion is a technique in which flaps of dura mater centered around the middle meningeal artery are inverted, encouraging the formation of a rich collateral blood supply. This procedure has been used in combination with encephaloduroarteriosynangiosis for more than 20 years at the authors' institution for the treatment of pediatric moyamoya disease. The objective of this study was to describe the clinical and radiographic outcomes for a cohort of consecutive pediatric moyamoya patients undergoing dural inversion.
\end{abstract}

METHODS Clinical and radiographic data on patients who had undergone dural inversion in the period from 1997 to 2016 were reviewed. Univariate and multivariate logistic regression and Kaplan-Meier analyses were performed to assess the risk of postoperative stroke, functional outcome, and the angiographic degree of revascularization.

RESULTS Dural inversion was performed on 169 hemispheres in 102 patients. Median follow-up was 4.3 years. Six patients (3.6\% of hemispheres) suffered postoperative ischemic or hemorrhagic stroke. Overall mortality was $1.0 \%$. Good postoperative neurological status (modified Rankin Scale [mRS] score $\leq 2$ ) was observed in 90 patients (88\%); preoperative and postoperative mRS scores showed significant improvement $(p<0.001)$. Eighty-six percent of hemispheres had Matsushima grade A or B revascularization. Younger age was associated with postoperative stroke and poor functional outcome. Patients with secondary moyamoya syndrome had a significantly worse radiographic outcome. The cumulative 5-year Kaplan-Meier risk for stroke was 6.4\%.

CONCLUSIONS Dural inversion is a useful technique of cerebral revascularization in pediatric moyamoya disease. A 20 -year experience demonstrates the safety and efficacy of this technique with a relatively low rate of postoperative stroke, good functional outcomes, and favorable angiographic results.

https://thejns.org/doi/abs/10.3171/2018.5.PEDS18163

KEYWORDS moyamoya; dural inversion; stroke; indirect revascularization; vascular disorders

$\mathrm{M}$ OYAMOYA disease is a progressive occlusive arteriopathy characterized by stenosis and occlusion of the internal carotid artery and its large branches. Over time, abnormal dilated vessels develop from internal carotid tributaries known as "moyamoya vessels," followed by the development of collateral blood supply from external carotid artery feeders. In childhood, moyamoya disease usually presents with ischemic events due to the progressive reduction of cerebral blood flow. Secondary moyamoya syndrome occurs in association with other medical conditions such as Down's syndrome, neurofibromatosis, sickle cell anemia, and cranial irradiation. ${ }^{23}$

Surgical intervention is the standard therapy for patients with moyamoya. Results from the International Pediatric Stroke Study confirm that children treated with surgical revascularization are less likely to have recurrent

ABBREVIATIONS CSF = cerebrospinal fluid; EDAS = encephaloduroarteriosynangiosis; $M C A=$ middle cerebral artery; $M M A=$ middle meningeal artery; $m R S=$ modified Rankin Scale; STA = superficial temporal artery.

SUBMITTED April 9, 2018. ACCEPTED May 25, 2018.

INCLUDE WHEN CITING Published online August 17, 2018; DOI: 10.3171/2018.5.PEDS18163.

${ }^{*}$ N.G. and S.L. contributed equally to this study and share first authorship. 
stroke than those treated medically. ${ }^{12,23,38}$ Various surgical procedures have been developed to facilitate revascularization of the ischemic brain. Direct anastomosis between the superficial temporal artery (STA) and middle cerebral artery (MCA) is less commonly applied to children given the small caliber of their STA ${ }^{7}$ as well as the need for temporary occlusion of an MCA branch required for anastomosis. Indirect revascularization exploits the neovascularization of ischemic brain with leptomeningeal collateral blood supply. Encephaloduroarteriosynangiosis (EDAS), created by Matsushima et al. in $1981,{ }^{26}$ consists of dissection and direct apposition of the STA to the surface of the brain. Variations of this procedure such as encephalomyosynangiosis and pial synangiosis have been explored. Capitalizing on the vascularity of dura mater with techniques such as burr holes has also been well described. ${ }^{28}$

In 1997, Dauser et al. described the technique of dural inversion in which large dural flaps centered around the middle meningeal artery (MMA) are created. The dural flaps are then inverted, allowing an increased surface area of contact between the vascularized outer dural layer and the ischemic brain. ${ }^{5}$ In the present study, we describe the treatment of pediatric moyamoya disease using dural inversion combined with EDAS in 169 consecutive surgeries over a 20 -year period at a single institution.

\section{Methods \\ Study Design}

Pediatric patients who had undergone revascularization for moyamoya disease or syndrome between 1997 and 2016 were identified from the database at our tertiary care academic hospital and were retrospectively reviewed according to the STROBE Statement guidelines. Patients were diagnosed with moyamoya based on clinical presentation and neuroimaging studies in concordance with standard diagnostic criteria. ${ }^{8}$ Patients with less than 6 months of postoperative follow-up were excluded; three patients were excluded on this basis. Preoperative angiographic findings were categorized into six stages based on the Suzuki grading system. ${ }^{35}$ Medical records were abstracted for demographics, symptomatology, radiographic results, surgical information, postoperative course, and long-term outcome. Approval was obtained from the Institutional Research Board of Baylor College of Medicine, and a waiver of patient consent was granted.

\section{Surgical Technique}

Revascularization is offered to patients with angiographic evidence of moyamoya who present with ischemia, hemorrhage, transient ischemic attack, or seizures. Contralateral revascularization is offered for patients with radiographic evidence of contralateral moyamoya (Suzuki stage $\geq$ II) even if asymptomatic. For patients with bilateral disease, surgery is first performed on the more affected hemisphere. Aspirin is administered to all patients preoperatively and is continued through the peri- and postoperative period, unless contraindicated (hemorrhage, coagulopathy).

The surgical technique of dural inversion has been described in detail previously. ${ }^{5}$ Briefly, the vertical incision enabling exposure of the STA is connected with a horizontal incision at the superior temporal line going anteriorly, allowing a frontoparietal bone flap to be turned and visualization of the MMA (Fig. 1A). The dura on either side of the MMA is incised to create two dural flaps, each on either side of the pedicled artery; dural incisions are stopped $2 \mathrm{~mm}$ short of the MMA to prevent twisting and occlusion (Fig. 1B). The arachnoid is opened simultaneously with the dura, as evidenced by cerebrospinal fluid (CSF) egress, thereby exposing the vascularized onlay tissues to growth factors of the CSF. The dural flaps are inverted, one flap passing above and one below the MMA without kinking the artery (Fig. 1C). The flaps of dura are loosely tacked into position. The bone flap is replaced with spaces in the craniotomy to allow passage of the STA.

Dural inversion is performed in conjunction with EDAS, except in cases in which the STA is too small to perform EDAS. There is no strict age cutoff at which EDAS is not performed, though we note that patients younger than 12 months have diminutive STAs and that the bone flap created based on the STA would provide relatively little surface area for the dural inversion. Thus, we have evolved to using 12 months as an age guideline below which we are less likely to perform EDAS in conjunction with the dural inversion. Preoperative angiography indicating an STA diameter less than $1 \mathrm{~mm}$ and the inability to insonate and map the course of the STA intraoperatively with Doppler ultrasound are also relative contraindications to EDAS.

\section{Follow-Up}

Postoperative angiography is performed at least 6 months following surgery. After the initial postoperative angiography, follow-up consists of yearly MRI, MRA, and clinical evaluation.

The following three clinical outcomes were evaluated: incidence of postoperative stroke including ischemic and hemorrhagic events, neurological status at the time of surgery and at follow-up according to the modified Rankin Scale (mRS), and degree of revascularization as graded by an independent neuroradiologist (M.P.) according to the Matsushima scale. The mRS is a 7-point grading scale ranging from 0 (no symptoms) to 6 (death); a score above 2 points indicates functional dependence. ${ }^{36}$ The Matsushima grading scale describes the extent of perfusion on postoperative angiograms and is divided into three grades: A (area perfused by revascularization $>2 / 3$ MCA territory), B (area perfused between $1 / 3$ and 2/3 MCA territory), and $\mathrm{C}$ (area perfused $<1 / 3$ MCA territory). ${ }^{27}$

\section{Statistical Analysis}

The association between clinical factors and outcome was assessed on univariate analysis using the two-sample t-test, chi-square test, and Fisher's exact test, as well as by multivariate logistic regression analysis. Adjusted odds ratios and $95 \%$ confidence intervals were calculated. Stroke risk was calculated using the Kaplan-Meier method, where start time was the time of surgery (if bilateral surgeries were performed, time of the first surgery) and event time was the time of postoperative ischemic or hemorrhagic 


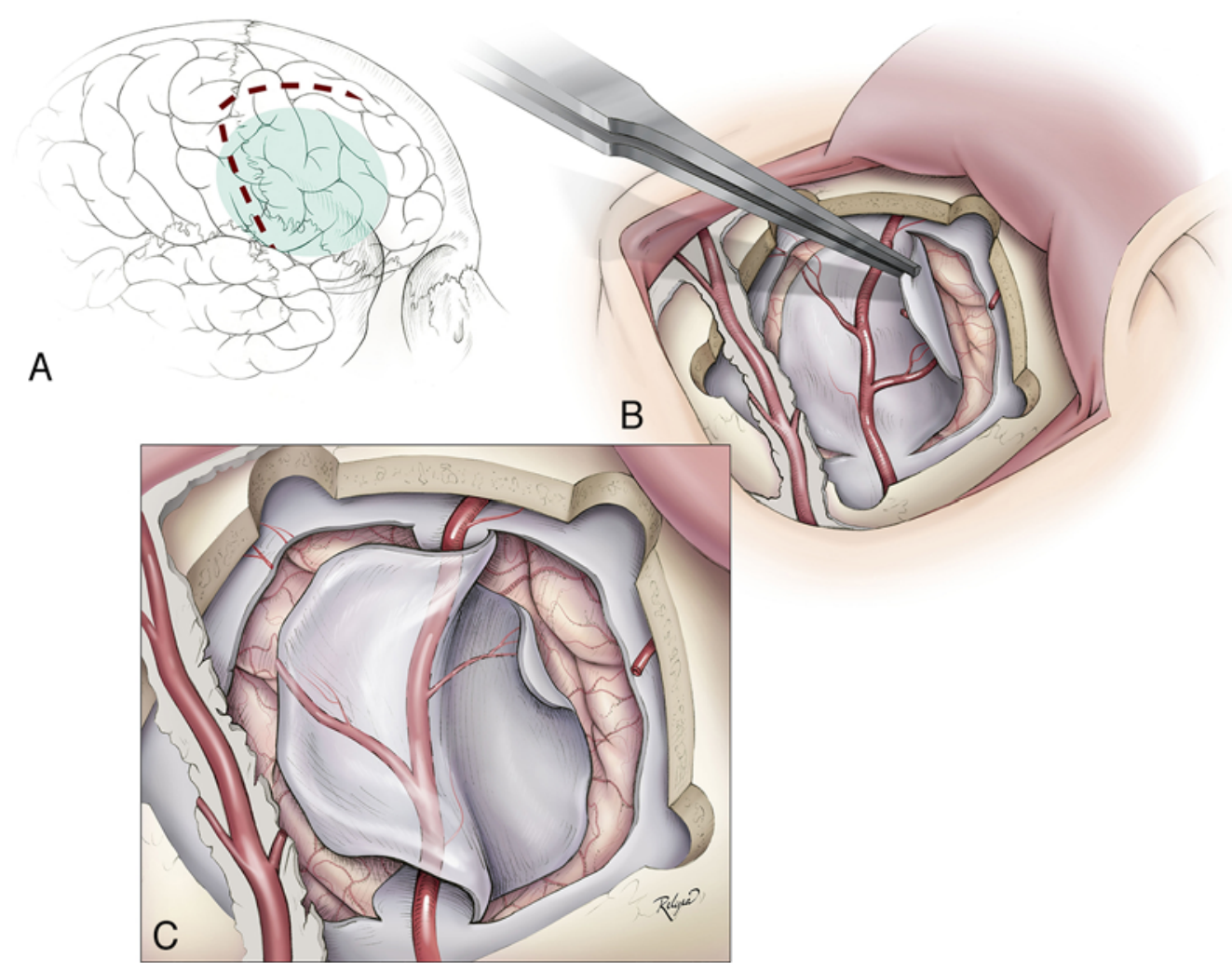

FIG. 1. A: Location and size of the frontoparietal bone flap is depicted in green. The dotted line indicates the horizontal skin incision that is connected with the vertical incision, exposing the STA. B: Dural flaps centered about the MMA are created, leaving a 2-mm cuff of dura around the artery. Encephaloduroarteriosynangiosis is simultaneously performed. C: The dural flaps are inverted to appose the outer vascular periosteal dural layer to ischemic brain, with care taken not to kink or occlude the artery. Copyright Sandi Lam. Published with permission. Figure is available in color online only.

stroke. All statistical analyses were conducted using Stata 14.2 (StataCorp). A p value $<0.05$ was considered significant.

\section{Results}

\section{Demographics and Clinical Information}

Between 1997 and 2016, a total of 102 patients underwent 169 revascularization surgeries for moyamoya disease; 67 patients had bilateral revascularization. Clinical and demographic results are summarized in Table 1. Fiftyfive percent of the patients were female. The demographic breakdown was as follows: $48 \%$ black/African American, $26.5 \%$ white, $17.6 \%$ Hispanic, and $8 \%$ other/unknown. Median age at the time of first surgery was 9.9 years. Moyamoya was considered secondary in 60 patients $(59 \%)$, most commonly due to sickle cell disease (42\%), cranial irradiation (8\%), and Down's syndrome (5\%). Preoperative Suzuki stages are shown in Table 1; this information was lacking in 16 hemispheres. The median preoperative Suzuki stage was III. Of 169 surgeries, 115 involved dural inversion with EDAS (68\%); the remaining 54 surgeries (32\%) involved dural inversion alone.

\section{Surgical Morbidity}

Clinically relevant ischemic and hemorrhagic complications are detailed in Table 2. Three patients suffered ischemic stroke within the surgical hemisphere within 30 days of surgery. One patient with Alagille syndrome and platelet dysfunction suffered postoperative intracerebral hemorrhage requiring craniotomy for evacuation. Two other patients presented with delayed ischemic events at 1 and 3 years postoperatively. One of these patients died from ischemic complications, which represents the only death related to surgery or stroke (mortality rate $1.0 \%$ ). This patient had progressive systemic vasculitis affecting multiple organs. Overall, the rate of postoperative stroke was 3.6\% per procedure (3 early ischemic events, 2 late ischemic events, 1 hemorrhage). Other complications within 30 days of surgery are as follows: three patients developed epidural or subdural hematoma at the surgical site requiring evacuation. Two patients developed chronic subdural hematomas requiring evacuation; both had underlying coagulopathy. One patient developed postoperative hydrocephalus requiring shunt placement. After follow-up angiography, one patient developed subarachnoid hemorrhage from rupture of an unrelated superior cerebellar artery aneurysm, which required ventriculostomy placement and endovascular coiling of the aneurysm; this patient made a full recovery. Eleven patients (6.5\% of procedures) experienced postoperative seizure, three suffered postoperative transient ischemic attack, two developed postoperative pneumonia, and one patient each had CSF leak, shunt infection, or wound dehiscence and resultant intracranial abscess. 
TABLE 1. Demographic and clinical data for 102 patients with moyamoya disease treated with dural inversion

\begin{tabular}{|c|c|c|}
\hline Parameter & No. & $\%$ \\
\hline Total no. of patients & 102 & \\
\hline Total no. of hemispheres & 169 & \\
\hline Median FU in yrs (range) & $4.3(0.5-16.4)$ & \\
\hline Median age at 1st surgery in yrs (range) & $9.9(0.3-23.8)$ & \\
\hline Female sex & 56 & 54.9 \\
\hline \multicolumn{3}{|l|}{ Ethnicity } \\
\hline Black or African American & 49 & 48.0 \\
\hline White & 27 & 26.5 \\
\hline Hispanic & 18 & 17.6 \\
\hline Other/unknown & 8 & 7.8 \\
\hline \multicolumn{3}{|l|}{ Etiology/associated findings } \\
\hline Sickle cell disease & 43 & 42.2 \\
\hline Idiopathic & 41 & 40.2 \\
\hline Radiation & 8 & 7.8 \\
\hline Down's syndrome & 5 & 4.9 \\
\hline NF1 & 1 & 1.0 \\
\hline ACTA2 mutation & 1 & 1.0 \\
\hline Alagille syndrome & 1 & 1.0 \\
\hline GUCY1A3 mutation & 1 & 1.0 \\
\hline Morning glory eye & 1 & 1.0 \\
\hline \multicolumn{3}{|l|}{ Presenting characteristic } \\
\hline Stroke & 83 & 81.4 \\
\hline TIAs w/o ischemia & 7 & 6.9 \\
\hline Intracranial hemorrhage & 5 & 4.9 \\
\hline Incidental & 3 & 2.9 \\
\hline Other & 2 & 2.0 \\
\hline \multicolumn{3}{|l|}{ Initial Suzuki stage* } \\
\hline 1 & 8 & 4.7 \\
\hline II & 28 & 16.6 \\
\hline III & 69 & 40.8 \\
\hline IV & 38 & 22.5 \\
\hline V & 9 & 5.3 \\
\hline $\mathrm{VI}$ & 1 & 0.6 \\
\hline \multicolumn{3}{|l|}{ Surgical procedure* } \\
\hline Dural inversion + EDAS & 115 & 68.0 \\
\hline Dural inversion only & 54 & 32.0 \\
\hline
\end{tabular}

EDAS = encephaloduroarteriosynangiosis; FU = follow-up; NF1 = neurofibromatosis type 1; TIA = transient ischemic attack.

* Listed separately for each operated hemisphere.

\section{Follow-Up}

Median follow-up was 4.3 years postoperatively (range $0.5-16.4$ years). Good postoperative neurological status at the last follow-up (mRS score $\leq 2$ ) was observed in 90 patients (88\%); mean preoperative and postoperative $\mathrm{mRS}$ scores were 1.8 and 0.9 , respectively ( $p<0.001$; Fig. 2 left). In 3 patients, the mRS scores worsened postoperatively; in the remaining $97 \%$ of patients, the mRS score was unchanged or improved (Fig. 2 right). Matsushima grades as determined by independent neuroradiologist review of postoperative cerebral angiograms were as follows (\% hemispheres): $68 \%$ grade $\mathrm{A}, 18 \%$ grade $\mathrm{B}$, and $14 \%$ grade $\mathrm{C}$.

\section{Outcomes Analysis}

Patients with secondary moyamoya syndrome were more likely to have a poor angiographic outcome (Matsushima grade $\mathrm{C}, \mathrm{p}=0.013$; for this analysis, we considered the worse score of the two sides when bilateral surgery was performed). Results of univariate analysis are depicted in Table 3. Younger age was associated with a postoperative stroke event as well as a poor functional status. Patients undergoing dural inversion alone were more likely to have a poor functional outcome; however, this finding was not borne out in the multivariate analysis. Results of multivariate analysis, listed in Tables 4 and 5, confirmed an increased likelihood of postoperative stroke in younger patients $(\mathrm{OR}=0.75, \mathrm{p}=0.042)$. The cumulative 5-year Kaplan-Meier risk of ischemic or hemorrhagic stroke was $6.4 \%$ (95\% CI 3\%-14\%). To determine whether there was a change in outcomes over the 20 -year period in which the surgical technique has been employed, a cohort from the first 8 years of its use (1997-2004) was compared to the most recent cohort (2010-2017); there were no statistically significant differences in stroke events, functional outcomes, or angiographic grade of revascularization.

\section{Discussion}

Indirect revascularization is widely accepted as the treatment of choice in children with moyamoya disease. . $^{12,32,34}$ Direct revascularization in pediatric patients is less common: recipient and donor vessels are smaller and operative times are longer than those for indirect methods, and the temporary clamping required for direct anastomosis risks the interruption of transcortical collaterals that are often seen in these patients. Although some have successfully employed the direct method in children older than 4 years, two large reviews of the pediatric literature failed to demonstrate the superiority of direct methods over indirect. ${ }^{10,38}$ The efficacy of indirect revascularization surgery in children in particular may be attributable to higher levels of growth factors in the CSF of children with moyamoya; $; 4$ there is also likely an age-associated angiogenic capability.

Numerous strategies facilitate indirect revascularization of the ischemic brain. For example, EDAS utilizes the angiogenic potential of the STA, which is directly apposed to the cortical surface. This procedure has been widely adopted because of its simplicity, avoidance of intraoperative arterial occlusion, and excellent postoperative results. ${ }^{26,32,34}$ In 1995 Adelson and Scott reported a technical modification to include opening the arachnoid and sewing the STA adventitia directly to the pia (pial synangiosis). ${ }^{1}$ Other indirect techniques have been explored including encephalomyosynangiosis (implantation of the temporalis muscle) ${ }_{1}^{15}$ omental transplantation, ${ }^{16}$ and multiple burr holes. ${ }^{33}$

The MMA has long been investigated for its potential to offer a collateral blood supply to the ischemic cortex..$^{29}$ On its own, the MMA forms a collateral blood supply to a limited extent. The outer periosteal layer of the dura con- 
TABLE 2. Postoperative stroke complications

\begin{tabular}{cclllll}
\hline Yr of Surgery & Age $(\mathrm{yrs})$ & \multicolumn{1}{c}{ Etiology } & Operation/Side & \multicolumn{1}{c}{ Complication (timing) } & \multicolumn{1}{c}{ Location } \\
\hline 2007 & 6 & Idiopathic & DI + EDAS/rt & Stroke (POD 11) & Rt ACA territory & Full recovery \\
\hline 2013 & 7 & Idiopathic & DI + EDAS/rt & Stroke (POD 1) & Rt parietal & Rt paresis \\
\hline 2014 & 1 & Idiopathic & DI/rt & Stroke (POD 7) & Rt parietal & Lt hand weakness \\
\hline 2014 & 2 & Alagille syndrome & DI/rt & ICH (POD 1) treated w/ craniotomy & Rt parietal, It convexity & Full recovery \\
\hline 2006 & 1 & Idiopathic & DI/lt & Stroke (1 yr postop) & Bilat diffuse infarcts & Death \\
\hline 2009 & 6 & Sickle cell & DI + EDAS/rt & Stroke (3 yrs postop) & Rt ACA territory & Full recovery \\
\hline
\end{tabular}

$\mathrm{ACA}=$ anterior cerebral artery; $\mathrm{DI}=$ dural inversion; $\mathrm{ICH}=$ intracranial hemorrhage; $\mathrm{POD}=$ postoperative day.

tains vessels supplied by the MMA; although this layer demonstrates angiogenic activity, neovascularization does not extend across the inner (meningeal) layer of the dura, creating a barrier between the internal and external carotid blood supply. ${ }^{5,17}$ Studies have shown that in some cases even simple burr holes and dural opening allow the ingrowth of collateral vessels from dural edges. ${ }^{28}$

In dural inversion, dural flaps centered around the MMA pedicle are inverted and apposed to the brain, allowing contact between the richly vascular periosteal dura and the cortical surface to facilitate cortical neovascularization from the MMA. This can be combined with procedures such as EDAS, allowing multiple arterial sources of revascularization. As compared to burr hole procedures, dural inversion increases the surface area of contact between the outer layer of dura and the cortex. While EDAS is limited to the anatomical distribution of this artery, the location of the dural flaps can be adjusted according to the angiographic region of ischemia; multiple flaps can be created to encompass a larger region. Dural flaps can be utilized even when the STA is diminutive, especially in young children. If the STA is not incorporated, a larger skin incision and craniotomy can be made in younger children, thereby allowing a larger surface area of dura to be inverted. Example preoperative and postoperative angiograms are featured in Fig. 3.

The largest series utilizing this technique was con- ducted by Zhao et al., who described 57 patients undergoing dural inversion in combination with multiple burr holes and periosteal synangiosis; these authors noted an improved functional status and successful angiographic neovascularization in most patients. ${ }^{41}$ Another study of 12 patients undergoing superselective angiography following indirect revascularization demonstrated greater angiographic contribution of the MMA than the STA in those who underwent dural inversion. ${ }^{21}$ McLaughlin and Martin described a variation on dural management in which a cruciate dural incision was created, the innermost dural layer was peeled away, and the arachnoid was opened to allow the ingrowth of MMA vessels. ${ }^{29}$ Another technique pioneered by Kashiwagi et al. advocates splitting the inner and outer dural layers and laying the split outer layer over the cortical surface. This technique demonstrated good results in their series of 18 patients. ${ }^{17}$

Since 1997 at our institution, dural inversion has been the preferred technique for revascularization in pediatric moyamoya. We perform EDAS in conjunction with dural inversion except in cases in which the STA is too small for EDAS. In the current study, we describe a consecutive series of 102 pediatric patients who underwent dural inversion for moyamoya disease at a single institution over a 20 -year span. The primary outcome of interest is the incidence of postoperative stroke. ${ }^{13,23}$ Previous studies have described a stroke risk of $32 \%$ to $38.9 \%{ }^{18}$ in patients with-
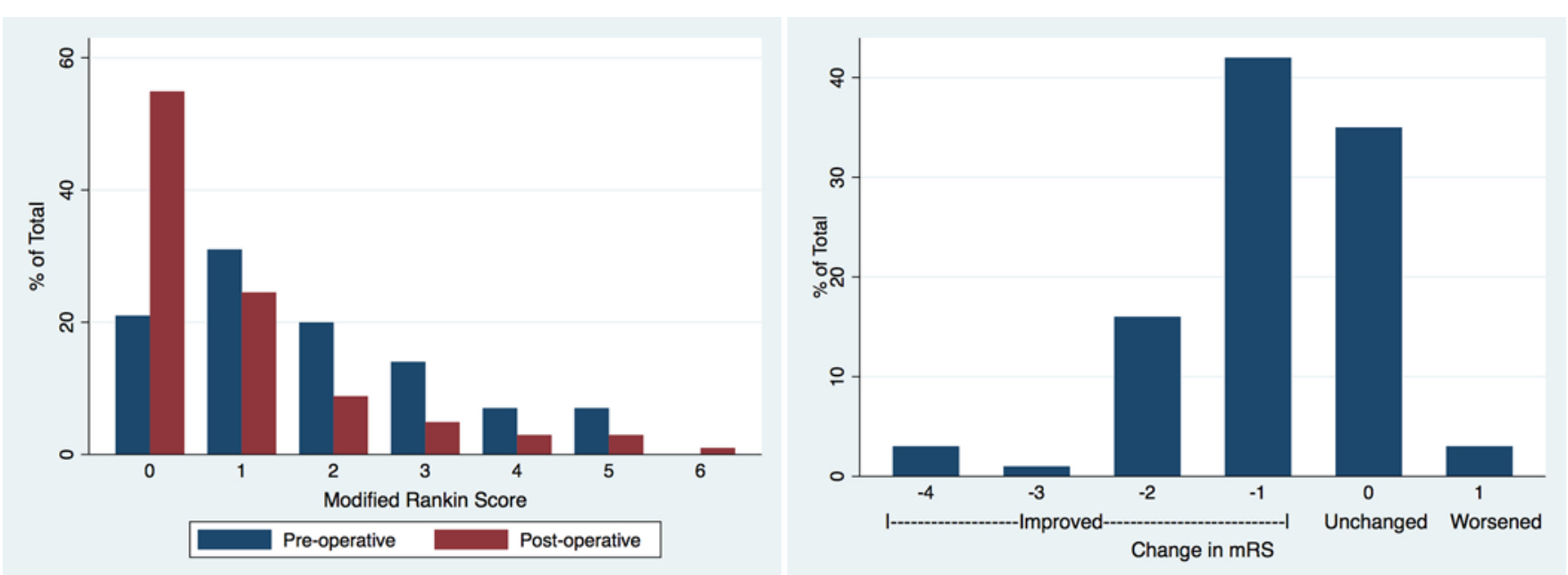

FIG. 2. Left: Bar graph demonstrating preoperative (b/ue) and postoperative (red) mRS scores. Right: Change in mRS scores from pre- to postoperatively. Figure is available in color online only. 
TABLE 3. Univariate analysis: risk factors for postoperative stroke and functional outcomes

\begin{tabular}{|c|c|c|c|c|c|c|}
\hline Parameter & $\begin{array}{l}\text { No Postop } \\
\text { Stroke }\end{array}$ & $\begin{array}{l}\text { Postop } \\
\text { Stroke }\end{array}$ & $p$ Value & $\begin{array}{l}\text { Good Functional Outcome } \\
\text { (mRS score } \leq 2)\end{array}$ & $\begin{array}{l}\text { Poor Functional Outcome } \\
\quad \text { (mRS score }>2)\end{array}$ & $p$ Value \\
\hline No. of patients & 96 & 6 & & 90 & 12 & \\
\hline Median age (yrs) & 9.2 & 3.7 & 0.008 & 9.2 & 6.4 & 0.035 \\
\hline \multicolumn{7}{|l|}{$\operatorname{Sex}(\%)$} \\
\hline Male & 93.5 & 6.5 & 0.804 & 93.5 & 6.5 & 0.136 \\
\hline Female & 94.6 & 5.4 & & 83.9 & 16.1 & \\
\hline Ethnicity (\%) & & & 0.547 & & & 0.498 \\
\hline Black or African American & 95.9 & 4.1 & & 89.8 & 10.2 & \\
\hline White & 88.9 & 11.1 & & 81.5 & 18.5 & \\
\hline Hispanic & 94.4 & 5.6 & & 88.9 & 11.1 & \\
\hline Other/unknown & 100.0 & 0.0 & & 100.0 & 0.0 & \\
\hline Etiology (\%) & & & 0.173 & & & 0.172 \\
\hline Secondary & 96.7 & 3.3 & & 91.8 & 8.2 & \\
\hline Idiopathic & 90.2 & 9.8 & & 82.9 & 17.1 & \\
\hline Presentation (\%) & & & 0.566 & & & 0.782 \\
\hline Stroke & 94.0 & 6.0 & & 86.8 & 13.2 & \\
\hline TIA w/o ischemia & 85.7 & 14.3 & & 100.0 & 0.0 & \\
\hline Intracranial hemorrhage & 100.0 & 0.0 & & 100.0 & 0.0 & \\
\hline Suzuki stage (\%) & & & 0.130 & & & 0.432 \\
\hline | or II & 100 & 0 & & 87.5 & 12.5 & \\
\hline III & 95.7 & 4.3 & & 93.5 & 6.5 & \\
\hline IV & 82.6 & 17.4 & & 82.6 & 17.4 & \\
\hline V or VI & 100.0 & 0.0 & & 87.5 & 12.5 & \\
\hline Surgical procedure (\%) & & & 0.915 & & & 0.032 \\
\hline Dural inversion + EDAS & 93.8 & 6.2 & & 92.9 & 7.1 & \\
\hline Dural inversion only & 94.3 & 5.7 & & 78.1 & 21.9 & \\
\hline
\end{tabular}

Boldface type indicates statistical significance.

out surgery. Furthermore, the natural history of moyamoya involves a slow neurological and cognitive decline over time. ${ }^{25}$ Thus, when analyzing the efficacy of this revascularization procedure, it is important to look at long-term functional outcome. In addition, radiographic revascularization is quantified using the Matsushima grading system.

Most large series of pediatric moyamoya patients come

TABLE 4. Multivariate analysis: risk of poor neurological outcome ( $\mathrm{mRS}$ score $>2$ )

\begin{tabular}{lccc}
\hline \multicolumn{1}{c}{ Parameter } & OR & $95 \% \mathrm{Cl}$ & $\mathrm{p} \mathrm{Value}$ \\
\hline Age & 0.91 & $0.79-1.05$ & 0.219 \\
\hline Sex & & & \\
\hline Male & Reference group & & \\
\hline Female & 2.31 & $0.55-9.75$ & 0.254 \\
\hline Etiology & & & \\
\hline Secondary & 0.70 & $0.18-2.82$ & 0.621 \\
\hline Idiopathic & Reference group & & \\
\hline Surgical procedure & & & \\
\hline Dural inversion + EDAS & Reference group & & \\
\hline Dural inversion only & 2.68 & $0.72-9.97$ & 0.142 \\
\hline
\end{tabular}

from Asia, where the incidence of moyamoya disease is known to be higher; there are few large pediatric series in the United States. ${ }^{2,34}$ The ethnic distribution in the present study demonstrated a higher percentage of African American (48\%) and Hispanic (18\%) patients than other United States series. ${ }^{4,12,37}$ There was only a slight female preponderance $(56 \%)$, which is also lower than the rates in other North American and European series. ${ }^{12,34}$ The majority of patients in our study had secondary moyamoya syndrome, most commonly due to sickle cell disease (42\%) or cranial radiation $(8 \%)$. Presenting symptomatology was overwhelmingly ischemic; hemorrhagic presentation in children is rare.

The incidence of postoperative ischemic or hemorrhagic

TABLE 5. Multivariate analysis: risk of postoperative stroke

\begin{tabular}{lccc}
\hline Parameter & OR & $95 \% \mathrm{Cl}$ & $\mathrm{p}$ Value \\
\hline Age & 0.75 & $0.57-0.99$ & $\mathbf{0 . 0 4 2}$ \\
\hline Etiology & & & \\
\hline Secondary & 0.77 & $0.11-5.51$ & 0.796 \\
\hline Idiopathic & Reference group & & \\
\hline
\end{tabular}

Boldface type indicates statistical significance. 

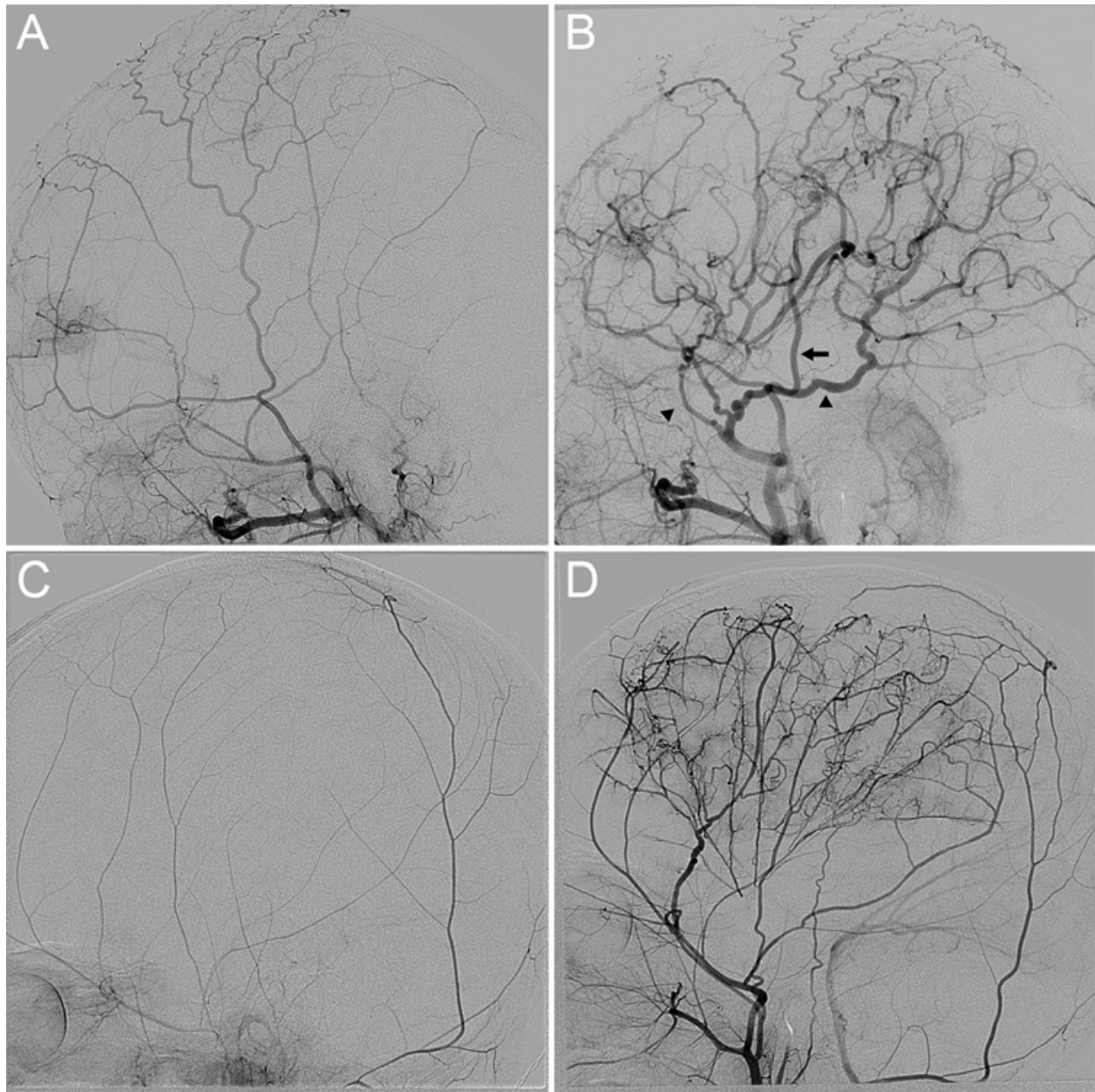

FIG. 3. Preoperative (A) and postoperative (B) lateral external carotid artery angiograms obtained in a 10 -year-old female who underwent bilateral dural inversion and EDAS. The preoperative angiogram demonstrates minimal external carotid artery collaterals. The postoperative angiogram demonstrates a dilated STA (arrow) and MMA (arrowheads) with extensive intracranial collateralization. Preoperative (C) and postoperative (D) lateral external carotid artery angiograms obtained in a 3-year-old female who underwent bilateral dural inversion. Her STA was not audible on Doppler bilaterally; therefore, we proceeded with dural inversion alone without EDAS, which allowed for a larger craniotomy and dural flaps, increasing the surface area of revascularization. The postoperative angiogram demonstrates exuberant intracranial collateralization from the MMA.

stroke was $3.6 \%$ per procedure, corresponding to a 5 -year Kaplan-Meier stroke risk of $6.4 \%$. There were three cases of stroke within 30 days of surgery, which is thought to be the most critical time for ischemia due to the theoretically delayed effect of indirect revascularization. Two patients presented with delayed stroke at 1 and 3 years postoperatively. One had attained Matsushima grade A revascularization bilaterally; he presented with anterior cerebral artery territory stroke and went on to make a full recovery. The other patient died of progressive diffuse multiorgan vasculitis, representing the only death in the series (mortality rate $1.0 \%$ ). Late-onset strokes have been reported up to 10 years following revascularization; however, the etiology is unclear. ${ }^{30}$ On univariate and multivariate analysis, postoperative stroke was correlated to younger age, an effect that has been noted by other groups. ${ }^{2,14}$ Other factors such as primary versus secondary moyamoya, presenting symptomatology, and angiographic grade did not correlate with postoperative stroke risk. Our results are comparable to those of other large series: Scott et al. reported a 30-day perioperative stroke rate of $7.7 \%$ in 143 pediatric patients undergoing pial synangiosis; ${ }^{34}$ Guzman et al. reported a 5 -year stroke or mortality rate of 5.5\% in 329 mixed adult and pediatric patients. ${ }^{12}$ Other large Asian pediatric series have reported a postoperative stroke rate of 3.4\%-13\% following mostly indirect revascularization procedures. ${ }^{3,19,30}$

Functional outcome was improved or unchanged in all but 3 patients in the present series; $88 \%$ of patients had a score of 0 (no symptoms) to 2 (mild neurological deficit) on the mRS at follow-up. Younger age and the use of dural inversion without EDAS were correlated with worse functional outcomes on univariate analysis; however, neither effect was borne out on multivariate analysis. There is some overlap with younger age and smaller STA that may contribute to these observations. The results from our study are comparable or favorable as compared to those of other large pediatric series, which report a good functional outcome in $80 \%-86 \%$ of patients. ${ }^{2,19,30,34}$ 
Although angiographic outcome has not necessarily been linked to clinical outcome, the degree of angiographic revascularization is an important postoperative measurement. In our study, $68 \%$ of the 169 cerebral hemispheres had Matsushima grade A revascularization; $18 \%$ had grade B. Scott et al. reported comparable results: $65 \%$ Matsushima grade A in 195 hemispheres following pial synangiosis. ${ }^{34}$ Bao et al. reported $43 \%$ Matsushima grade A following EDAS in 182 hemispheres. ${ }^{2}$

Secondary moyamoya syndrome exists in association with other systemic processes such as sickle cell anemia, Down's syndrome, and cranial irradiation. In these patients, the mechanism of moyamoya disease is believed to be different from that in the idiopathic form. In sickle cell disease (representing $42 \%$ of patients in the present series), recurrent endothelial injury from adhesion of sickle cells is thought to precipitate arterial stenosis; this process may be present in up to $43 \%$ of patients with sickle cell disease. ${ }^{6}$ Although many patients are treated with chronic transfusion therapy, over half may nevertheless incur further cerebral ischemia. ${ }^{11}$ Several studies have demonstrated the efficacy of surgical revascularization over the best medical management in reducing stroke risk in this population. ${ }^{39,40}$ In our series, $56 \%$ of patients with sickle cell disease had Matsushima grade A and 25\% attained Matsushima grade $\mathrm{B}$, which are poorer results than in the idiopathic subset; however, $93 \%$ of the patients with sickle cell disease achieved a favorable functional outcome. Following radiation exposure, endothelial injury, adventitial inflammation, and perivascular fibrosis cause morphological changes and vascular remodeling that can lead to moyamoya-like changes. $^{22,31}$ These have been treated with revascularization surgery by some, ${ }^{20,22}$ though data regarding outcomes are limited. In our series, $45 \%$ of patients with prior irradiation had poor angiographic revascularization (Matsushima grade $\mathrm{C}$ ), although a favorable functional outcome was seen in $88 \%$. Although a poor angiographic outcome in our series was correlated with secondary moyamoya $(p$ $=0.013$ ), these patients did not incur a higher stroke risk or poorer functional outcomes. This finding supports the hypothesis that the secondary moyamoya phenomenon is pathophysiologically different from idiopathic moyamoya. Angiographic revascularization may not be as robust; however, favorable clinical outcomes advocate for continued application of revascularization surgery in these patients.

Over the 20-year period during which dural inversion has been utilized at our institution, the surgical technique has not changed considerably. An analysis of outcomes from the first 8 years (1997-2004) compared to those from the most recent 8 years (2010-2017) revealed no difference in the stroke rate, functional outcomes, or angiographic revascularization. However, we note that surgical decisionmaking did evolve during this time period after early results demonstrated favorable revascularization originating from the MMA. This observation led us to consider dural inversion even in young children (age $<12$ months) in whom the STA was diminutive in size and EDAS was less feasible, as the MMA and dural arteries would likely provide sufficient revascularization from dural inversion alone in appropriate surgical candidates.

Limitations of this study include those inherent to ret- rospective chart reviews. In addition, there was no control group with which to compare outcomes and verify the beneficial effects of dural inversion. However, loss to follow-up was low, and the sample size compares favorably to those in other series. The use of objective radiographic and clinical outcome measures enables reproducible communication of results. Independent neuroradiology evaluation endeavored to minimize bias in the interpretation of angiographic results. Given the known limitations of single-institution clinical series, future directions might include multicenter collaboration to increase sample size and investigate subsets of patient phenotypes, clinical features, surgical techniques, and perioperative management.

\section{Conclusions}

Surgical revascularization has been established as the treatment of choice in moyamoya disease to prevent further stroke and neurological decline. Dural inversion, often in combination with EDAS, has been the preferred surgical technique at our institution for 20 years. Dural inversion is an accessible technique even when the STA is not available for EDAS. In our cohort of 102 patients and 169 cerebral hemispheres, the 5-year risk of stroke or hemorrhage postoperatively was $6.4 \%$ with a favorable functional outcome in $88 \%$ of patients. Our experience with this procedure demonstrates its safety and efficacy, with a low rate of postoperative stroke events, good functional outcomes, and favorable angiographic results.

\section{References}

1. Adelson PD, Scott RM: Pial synangiosis for moyamoya syndrome in children. Pediatr Neurosurg 23:26-33, 1995

2. Bao XY, Duan L, Yang WZ, Li DS, Sun WJ, Zhang ZS, et al: Clinical features, surgical treatment, and long-term outcome in pediatric patients with moyamoya disease in China. Cerebrovasc Dis 39:75-81, 2015

3. Blauwblomme T, Mathon B, Naggara O, Kossorotoff M, Bourgeois M, Puget S, et al: Long-term outcome after multiple burr hole surgery in children with moyamoya angiopathy: a single-center experience in 108 hemispheres. Neurosurgery 80:950-956, 2017

4. Chiu D, Shedden P, Bratina P, Grotta JC: Clinical features of moyamoya disease in the United States. Stroke 29:13471351, 1998

5. Dauser RC, Tuite GF, McCluggage CW: Dural inversion procedure for moyamoya disease. Technical note. J Neurosurg 86:719-723, 1997

6. Dobson SR, Holden KR, Nietert PJ, Cure JK, Laver JH, Disco D, et al: Moyamoya syndrome in childhood sickle cell disease: a predictive factor for recurrent cerebrovascular events. Blood 99:3144-3150, 2002

7. Fukui M: Current state of study on moyamoya disease in Japan. Surg Neurol 47:138-143, 1997

8. Fukui M: Guidelines for the diagnosis and treatment of spontaneous occlusion of the circle of Willis ("moyamoya" disease). Clin Neurol Neurosurg 99 (Suppl 2):S238-S240, 1997

9. Fullerton HJ, Wintermark M, Hills NK, Dowling MM, Tan M, Rafay MF, et al: Risk of recurrent arterial ischemic stroke in childhood: a prospective international study. Stroke 47:53-59, 2016

10. Fung LWE, Thompson D, Ganesan V: Revascularisation surgery for paediatric moyamoya: a review of the literature. Childs Nerv Syst 21:358-364, 2005 
11. Griessenauer CJ, Lebensburger JD, Chua MH, Fisher WS III, Hilliard L, Bemrich-Stolz CJ, et al: Encephaloduroarteriosynangiosis and encephalomyoarteriosynangiosis for treatment of moyamoya syndrome in pediatric patients with sickle cell disease. J Neurosurg Pediatr 16:64-73, 2015

12. Guzman R, Lee M, Achrol A, Bell-Stephens T, Kelly M, Do $\mathrm{HM}$, et al: Clinical outcome after 450 revascularization procedures for moyamoya disease. Clinical article. J Neurosurg 111:927-935, 2009

13. Hallemeier CL, Rich KM, Grubb RL Jr, Chicoine MR, Moran CJ, Cross DT III, et al: Clinical features and outcome in North American adults with moyamoya phenomenon. Stroke 37:1490-1496, 2006

14. Jackson EM, Lin N, Manjila S, Scott RM, Smith ER: Pial synangiosis in patients with moyamoya younger than 2 years of age. J Neurosurg Pediatr 13:420-425, 2014

15. Karasawa J, Kikuchi H, Furuse S, Sakaki T, Yoshida Y: A surgical treatment of "moyamoya" disease "encephalo-myo synangiosis". Neurol Med Chir (Tokyo) 17:29-37, 1977

16. Karasawa J, Touho H, Ohnishi H, Miyamoto S, Kikuchi H: Cerebral revascularization using omental transplantation for childhood moyamoya disease. J Neurosurg 79:192-196, 1993

17. Kashiwagi S, Kato S, Yasuhara S, Wakuta Y, Yamashita T, Ito H: Use of a split dura for revascularization of ischemic hemispheres in moyamoya disease. J Neurosurg 85:380 383, 1996

18. Kelly ME, Bell-Stephens TE, Marks MP, Do HM, Steinberg GK: Progression of unilateral moyamoya disease: A clinical series. Cerebrovasc Dis 22:109-115, 2006

19. Kim SK, Cho BK, Phi JH, Lee JY, Chae JH, Kim KJ, et al: Pediatric moyamoya disease: an analysis of 410 consecutive cases. Ann Neurol 68:92-101, 2010

20. Kim TG, Kim DS, Chung SS, Choi JU: Moyamoya syndrome after radiation therapy: case reports. Pediatr Neurosurg 47:138-142, 2011

21. King JAJ, Armstrong D, Vachhrajani S, Dirks PB: Relative contributions of the middle meningeal artery and superficial temporal artery in revascularization surgery for moyamoya syndrome in children: the results of superselective angiography. J Neurosurg Pediatr 5:184-189, 2010

22. Lee HS, Seol HJ, Kong DS, Shin HJ: Moyamoya syndrome precipitated by cranial irradiation for craniopharyngioma in children. J Korean Neurosurg Soc 50:535-537, 2011

23. Lee S, Rivkin MJ, Kirton A, deVeber G, Elbers J: Moyamoya disease in children: results from the International Pediatric Stroke Study. J Child Neurol 32:924-929, 2017

24. Malek AM, Connors S, Robertson RL, Folkman J, Scott RM: Elevation of cerebrospinal fluid levels of basic fibroblast growth factor in moyamoya and central nervous system disorders. Pediatr Neurosurg 27:182-189, 1997

25. Matsushima Y, Aoyagi M, Nariai T, Takada Y, Hirakawa K: Long-term intelligence outcome of post-encephalo-duro-arterio-synangiosis childhood moyamoya patients. Clin Neurol Neurosurg 99 (Suppl 2):S147-S150, 1997

26. Matsushima Y, Fukai N, Tanaka K, Tsuruoka S, Inaba Y, Aoyagi M, et al: A new surgical treatment of moyamoya disease in children: a preliminary report. Surg Neurol 15:313320,1981

27. Matsushima Y, Inaba Y: Moyamoya disease in children and its surgical treatment. Introduction of a new surgical procedure and its follow-up angiograms. Childs Brain 11:155170,1984

28. McLaughlin N, Martin NA: Effectiveness of burr holes for indirect revascularization in patients with moyamoya disease-a review of the literature. World Neurosurg 81:91-98, 2014

29. McLaughlin N, Martin NA: Meningeal management for opti- mal revascularization from middle meningeal artery. $\mathbf{J}$ Neurosurg 118:104-108, 2013

30. Mukawa M, Nariai T, Matsushima Y, Tanaka Y, Inaji M, Maehara T, et al: Long-term follow-up of surgically treated juvenile patients with Moyamoya disease. J Neurosurg Pediatr 10:451-456, 2012

31. Murphy ES, Xie H, Merchant TE, Yu JS, Chao ST, Suh JH: Review of cranial radiotherapy-induced vasculopathy. J Neurooncol 122:421-429, 2015

32. Park SE, Kim JS, Park EK, Shim KW, Kim DS: Direct versus indirect revascularization in the treatment of moyamoya disease. J Neurosurg [epub ahead of print October 27, 2017; DOI: $10.3171 / 2017.5 . J N S 17353]$

33. Sainte-Rose C, Oliveira R, Puget S, Beni-Adani L, Boddaert $\mathrm{N}$, Thorne J, et al: Multiple bur hole surgery for the treatment of moyamoya disease in children. J Neurosurg 105 (6 Suppl):437-443, 2006

34. Scott RM, Smith JL, Robertson RL, Madsen JR, Soriano SG, Rockoff MA: Long-term outcome in children with moyamoya syndrome after cranial revascularization by pial synangiosis. J Neurosurg 100 (2 Suppl Pediatrics):142-149, 2004

35. Suzuki J, Takaku A: Cerebrovascular "moyamoya" disease. Disease showing abnormal net-like vessels in base of brain. Arch Neurol 20:288-299, 1969

36. van Swieten JC, Koudstaal PJ, Visser MC, Schouten HJ, van Gijn J: Interobserver agreement for the assessment of handicap in stroke patients. Stroke 19:604-607, 1988

37. Uchino K, Johnston SC, Becker KJ, Tirschwell DL: Moyamoya disease in Washington State and California. Neurology 65:956-958, 2005

38. Veeravagu A, Guzman R, Patil CG, Hou LC, Lee M, Steinberg GK: Moyamoya disease in pediatric patients: outcomes of neurosurgical interventions. Neurosurg Focus 24(2):E16, 2008

39. Yang W, Porras JL, Xu R, Garzon-Muvdi T, Caplan JM, Colby GP, et al: Effectiveness of surgical revascularization for stroke prevention in pediatric patients with sickle cell disease and moyamoya syndrome. Neurosurgery 63 (Suppl 1):178, 2016 (Abstract)

40. Yang W, Xu R, Porras JL, Takemoto CM, Khalid S, GarzonMuvdi T, et al: Effectiveness of surgical revascularization for stroke prevention in pediatric patients with sickle cell disease and moyamoya syndrome. J Neurosurg Pediatr 20:232-238, 2017

41. Zhao X, Wang C, Ji Y, Han C, Wang M: Therapeutic effect of multiple burr hole operation combined with dural inversion and periosteal synangiosis for moyamoya disease. Br J Neurosurg 29:811-817, 2015

\section{Disclosures}

The authors report no conflict of interest concerning the materials or methods used in this study or the findings specified in this paper.

\section{Author Contributions}

Conception and design: Lam, Gadgil, Pyarali, Paldino, Dauser. Acquisition of data: Lam, Gadgil, Pyarali, Paldino, Dauser. Analysis and interpretation of data: Lam, Gadgil, Paldino, Pan, Dauser. Drafting the article: Gadgil. Critically revising the article: Lam, Gadgil, Pyarali, Paldino, Dauser. Reviewed submitted version of manuscript: Lam, Gadgil. Statistical analysis: Gadgil, Pan.

\section{Correspondence}

Sandi Lam: Texas Children's Hospital, Baylor College of Medicine, Houston, TX. sklam@texaschildrens.org. 